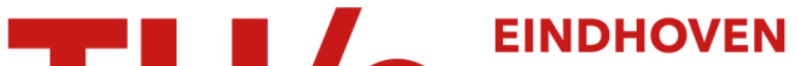

\section{Vesicles under simple shear flow : elucidating the role of the relevant control parameters}

Citation for published version (APA):

Kaoui, B., Farutin, A., \& Misbah, C. (2009). Vesicles under simple shear flow : elucidating the role of the relevant control parameters. Physical Review E - Statistical, Nonlinear, and Soft Matter Physics, 80(6), 061905-1/11.

[061905]. https://doi.org/10.1103/PhysRevE.80.061905

DOI:

10.1103/PhysRevE.80.061905

Document status and date:

Published: 01/01/2009

\section{Document Version:}

Publisher's PDF, also known as Version of Record (includes final page, issue and volume numbers)

\section{Please check the document version of this publication:}

- A submitted manuscript is the version of the article upon submission and before peer-review. There can be important differences between the submitted version and the official published version of record. People interested in the research are advised to contact the author for the final version of the publication, or visit the $\mathrm{DOI}$ to the publisher's website.

- The final author version and the galley proof are versions of the publication after peer review.

- The final published version features the final layout of the paper including the volume, issue and page numbers.

Link to publication

\section{General rights}

Copyright and moral rights for the publications made accessible in the public portal are retained by the authors and/or other copyright owners and it is a condition of accessing publications that users recognise and abide by the legal requirements associated with these rights.

- Users may download and print one copy of any publication from the public portal for the purpose of private study or research.

- You may not further distribute the material or use it for any profit-making activity or commercial gain

- You may freely distribute the URL identifying the publication in the public portal.

If the publication is distributed under the terms of Article 25fa of the Dutch Copyright Act, indicated by the "Taverne" license above, please follow below link for the End User Agreement:

www.tue.nl/taverne

Take down policy

If you believe that this document breaches copyright please contact us at:

openaccess@tue.nl

providing details and we will investigate your claim. 


\title{
Vesicles under simple shear flow: Elucidating the role of relevant control parameters
}

\author{
Badr Kaoui, ${ }^{1,2}$ Alexander Farutin, ${ }^{1}$ and Chaouqi Misbah ${ }^{1, *}$ \\ ${ }^{1}$ Laboratoire de Spectrométrie Physique, CNRS-Université Joseph Fourier/UMR 5588, \\ Boîte Postale 87, F-38402 Saint-Martin d'Hères Cedex, France \\ ${ }^{2}$ Faculté des Sciences Ben M'Sik, Laboratoire de Physique de la Matière Condensée, \\ Université Hassan II-Mohammedia, Boîte Postale 7955, 20800 Casablanca, Morocco
}

(Received 21 April 2009; revised manuscript received 13 August 2009; published 9 December 2009)

\begin{abstract}
The dynamics of vesicles under shear flow are carefully analyzed in the regime of a small vesicle excess area relative to a sphere. This regime corresponds to the quasispherical limit, for which several groups have analytically extracted simple nonlinear differential equations. Under shear flow, vesicles are known to exhibit three types of motion: (i) tank-treading (TT): the vesicle assumes a steady inclination angle with respect to the flow direction, while its membrane undergoes a tank-treading motion, (ii) tumbling (TB), and (iii) vacillatingbreathing (VB): the vesicle main axis oscillates about the flow direction, whereas the overall shape undergoes a breathinglike motion. The region of existence for each regime depends on material and control parameters. The whole set of parameters can be cast into three dimensionless control parameters: (i) the viscosity ratio between the internal and external fluid, $\lambda$, (ii) the excess area relative to a sphere (this parameter measures the degree of the vesicle deflation), $\Delta$, and (iii) the capillary number (the ratio between the vesicle relaxation time toward its equilibrium shape after cessation of the flow and the flow time scale, which is the inverse shear rate), Ca. Recent studies [Danker et al., Phys. Rev. E 76, 041905 (2007)] have focused on the shape of the phase diagram (representing the TT, TB, and VB regimes in the $\mathrm{Ca}-\lambda$ plane). In this paper, the physical quantities are analyzed in detail and attention is brought to features that are essential for future experimental studies. It is shown that the boundaries delimiting different dynamical regimes (TT, TB, and VB) in parameter space depend on the three dimensionless control parameters, in contrast with a recent study [V. V. Lebedev et al., Phys. Rev. Lett. 99, 218101 (2007)] where it is claimed that only two parameters are relevant. Consideration of the amplitude of oscillation (of the vesicle orientation angle and its shape deformation) in the VB mode reveals an even more significant dependence on the three parameters. It is also shown that the inclination angle in the TT regime significantly depends on the shear rate $(\mathrm{Ca})$, which runs contrary to common belief. Finally, we show that the TB and VB periods are quite insensitive to $\mathrm{Ca}$, in marked contrast with a recent study [H. Noguchi and G. Gompper, Phys. Rev. Lett. 98, 128103 (2007)].
\end{abstract}

DOI: 10.1103/PhysRevE.80.061905

PACS number(s): 87.16.D-, 47.63.-b, 73.43.Cd, 83.50.-v

\section{INTRODUCTION}

Vesicles are closed phospholipid membranes suspended in an aqueous solution $[1,2]$. They continue to gather increasing interest in different disciplines ranging from biology to mathematics. The vesicles that are the focus of this investigation are usually called "giant vesicles," and have a typical size in the 10-100 $\mu \mathrm{m}$ range (the word "giant" is used in reference to vesicles in the cytoplasm which are in the 100 nanometers range). Vesicles that are made of pure bilayer membranes mimic some features of the red blood cell (RBC), such as its equilibrium biconcave shape, tank-treading (TT) (see below), and tumbling (TB) under shear flow. Thus, vesicles have gained popularity as model systems for understanding RBC mechanics. Recent studies have highlighted that the unique mechanical properties of the lipid bilayer membrane, such as its fluidity, incompressibility, and resistance to bending, give rise to a number of fascinating nonequilibrium features of the vesicle and of RBC dynamics.

Vesicle dynamics under simple shear flow have been the subject of extensive theoretical and experimental studies

\footnotetext{
*chaouqi.misbah@ujf-grenoble.fr
}

[3-19]. It is known that vesicles under shear flow exhibit three different types of dynamical regimes. The most classical one is the TT mode (the vesicle assumes a steady inclination angle and a fixed shape, while the fluid membrane undergoes a tank-tread-like motion). This regime has also been observed for RBC [20].

Since this is a quickly advancing field, a brief review will first be presented. The TT of vesicles was analyzed by Keller and Skalak [3] (KS) (these authors actually considered RBCs), and later by Kraus et al. [4], using three-dimensional (3D) numerical techniques based on the boundary integral formulation. It was shown by KS that the inclination angle, $\Psi$ (see Fig. 1 for definition of the angle) of the vesicle main axis with respect to the flow is equal to $\pi / 4$ in the limiting case of a sphere, and decreases with the degree of the vesicle deflation. The full numerical solution [4] confirmed the analysis of KS. Seifert [6] later analyzed the TT regime in the quasispherical regime.

KS further showed that the TT regime exists within a certain range of control parameters. To focus the discussion, the three dimensionless parameters that enter the vesicle problem under a shear flow are:

(a) the excess area as defined by 


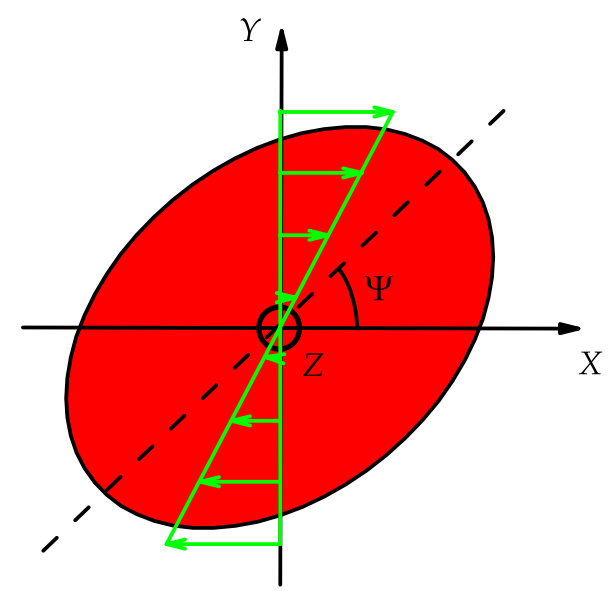

FIG. 1. (Color online) A schematic view of a vesicle in a shear flow, which shows the definition of the inclination angle in a linear shear flow. The arrows indicate the velocity field associated with a linear shear flow.

$$
\Delta=\frac{A-4 \pi R_{0}^{2}}{R_{0}^{2}},
$$

where $A$ is the vesicle area and $R_{0}$ is the radius of a sphere having the same volume as the vesicle. $\Delta=0$ for a sphere, and $\Delta>0$ otherwise. For human RBCs, $\Delta \simeq 5$. The available experimental data on vesicles have typically explored the range $\Delta=0.1-2$.

(b) The ratio between the viscosities of the internal $\left(\eta_{\text {int }}\right)$ and the external $\left(\eta_{\text {ext }}\right)$ fluids

$$
\lambda=\frac{\eta_{\text {int }}}{\eta_{\text {ext }}} .
$$

For a human $\mathrm{RBC}$ in vivo, $\lambda \simeq 5-6$. Laboratory experiments have explored the range of 0.1-20 both for vesicles and RBCs while varying the temperature and/or adding polymers inside the vesicle or in the suspending medium $[12,13,18]$.

(c) The capillary number, which is defined as the ratio between two characteristic time scales, the shearing time, $\tau_{\text {flow }}=1 / \gamma$, where $\gamma$ is the shear rate, and the typical time needed for a vesicle to attain its equilibrium shape after cessation of the flow, $\tau_{\text {shape }}=\eta_{\mathrm{ext}} R_{0}^{3} / \kappa$

$$
\mathrm{Ca}=\frac{\tau_{\text {shape }}}{\tau_{\text {flow }}}=\frac{\eta_{\text {ext }} \gamma R_{0}^{3}}{\kappa},
$$

where $\kappa$ is the membrane bending rigidity. Typical values from experimental investigations of vesicles lie roughly in the range $\mathrm{Ca} \sim 1-10^{3}[12,13]$.

$\mathrm{KS}$ showed that the TT regime exists below a critical value of $\lambda$, denoted $\lambda_{c}$, which depends on $\Delta$ (see Fig. 2). The inclination angle decreases with $\Delta$. Neither the inclination angle, nor the TT-TB boundary, have been found to depend on $\mathrm{Ca}$ (or shear rate) in the KS theory or in the numerical simulations $[3,4]$. It will be shown in this paper that a significant dependence on Ca may be exhibited.

KS showed that beyond $\lambda_{c}$, the TT solution ceases to exist and the vesicle undergoes TB. KS assumed a shape preserving solution (i.e., the vesicle maintains the same shape dur-

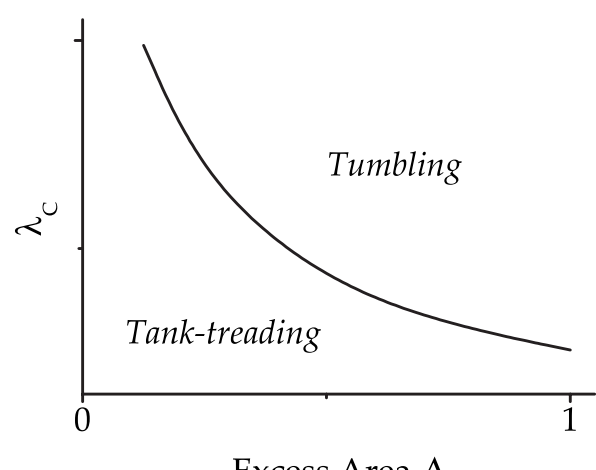

FIG. 2. A schematic view of the boundary $\lambda_{c}(\Delta)$ between TT and TB in the plane of the two control parameters, the viscosity ratio $\lambda$ and the vesicle excess area $\Delta . \Delta=0$ corresponds to a sphere where $\lambda_{c}$ is expected to diverge according to the KS theory. Note that the parameter $\mathrm{Ca}$ does not show up in the KS theory because the shape is fixed.

ing tumbling). The transition from TT to TB was subsequently numerically analyzed $[7,8]$ for two-dimensional (2D) vesicles (with no shape constraints, unlike KS theory) by means of the boundary integral formulation and phase-field models. It was been noted that the bifurcation from TT to TB is of saddle-node type [7]. The overall picture from the 2D numerical study [7] was found to be consistent with the KS analysis [3]. Figure 2 shows a schematic of the phase diagram. The TT motion has been analyzed experimentally by Kantsler and Steinberg [11] and the transition to TB by Mader et al. [13].

A regime has been revealed by recent research. A theoretical study [14] performed in the quasispherical regime has reported new dynamics, referred to as vacillating-breathing (VB) (later also described as trembling or swinging): the vesicle main axis oscillates about the flow direction, and the shape undergoes a breathinglike motion. In that work, it was shown that the VB motion coexists with TB. A movie shows the two regimes, VB and TB [21]. Which motion prevails depends on the initial conditions. This theory has truncated the expansion of the evolution equations about a spherical shape to leading order. As a consequence, the membrane bending rigidity (or $\mathrm{Ca}$ ) scaled out from the evolution equations, and only $\lambda$ and $\Delta$ remained. Later studies by Noguchi and Gompper [22], Lebedev et al. [16], and Danker et al. [23] continued the analysis to higher orders in the deviation from a sphere. Their main outcome is that the bending rigidity (or $\mathrm{Ca}$ ) shows up in the higher order expansion. A common result from these three investigations [[16,22,23]], is that the phase diagram in the $\mathrm{Ca}-\lambda$ plane has the qualitative form shown in Fig. 3. The careful analysis reported in this paper reveals, however, many important hidden differences.

The analysis of Noguchi and Gompper [22] regarding the dynamics of vesicle deformation was based on the work Ref. [14] supplemented with higher order contributions in the bending energy, and the KS theory [3] for the inclination angle. Lebedev et al. [16] followed a similar analysis as in [14], in which higher order contributions in the deviation from a sphere were partially included (the next higher order term was only added to the bending force, not the corre- 


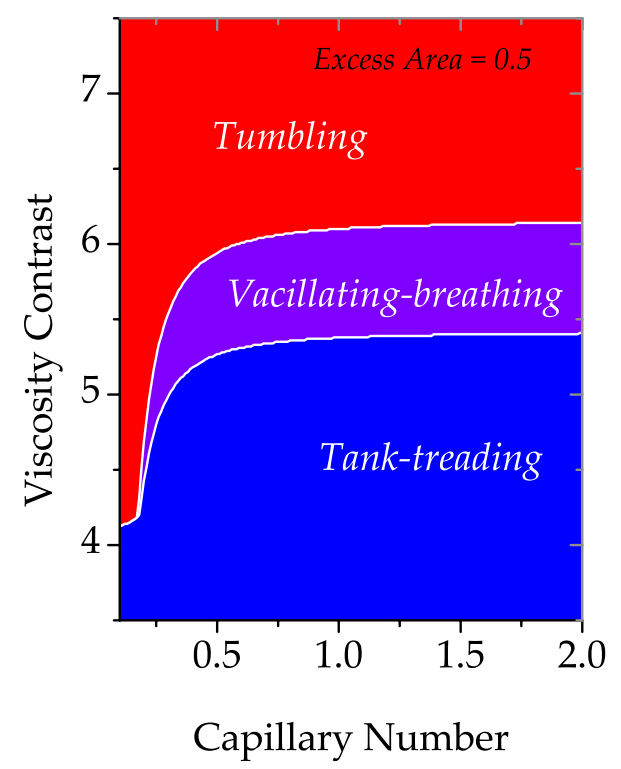

FIG. 3. (Color online) A typical phase diagram representing the three regimes: TT, VB, and TB in the $(\lambda-\mathrm{Ca})$ plane. This is the same phase diagram obtained in the present paper for $\Delta=0.5$.

sponding hydrodynamical response). Danker et al. [23] treated the higher order contribution consistently (i.e., they included the higher order terms to the bending energy and to the hydrodynamic response). Lebedev et al. [16] concluded that the dynamics of vesicles depend only on two independent control parameters defined as

$$
S=\frac{7 \sqrt{3} \pi}{9} \frac{\mathrm{Ca}}{\Delta}, \quad \Lambda=\frac{1}{240} \sqrt{\frac{30}{\pi}}(23 \lambda+32) \sqrt{\Delta} .
$$

This is in contradiction with the consistent theory of Danker et al. [23], according to which three control parameters are essential for the vesicle dynamics. This investigation revealed several features by following this theory.

The main findings of this work are: (i) the details of how the phase diagram (Fig. 3) describing regions of existence of the three regimes (TT, TB, and VB) depends on the three essential control parameters $(\mathrm{Ca}, \lambda, \Delta)$ (or alternatively $S, \Lambda$, and $\Delta$ ) are reported; (ii) in the TT regime, the inclination angle may vary with the shear rate (or $\mathrm{Ca}$ ), which has not been previously revealed; (iii) the period of the TB and VB modes as a function of the three relevant control parameters are described, which differ from the results of Noguchi and Gompper [22]. More precisely, a weak dependence (not more than 10-20\%) of the period was found as a function of $\mathrm{Ca}$, which is at least ten times smaller than that found by Noguchi and Gompper [22]; (iv) it is shown that the amplitude of oscillation of the main axis of the vesicle in the VB mode strongly depends on the excess area when $S$ and $\Lambda$ are constant. The change can vary by up to $200 \%$, however, the theory of Lebedev et al. [16] predicts no variation at all.

The present results should be useful for any future attempts to analyze experimental data. There have been only a few experimental results in which VB has been discussed $[12,13]$. It seems that an early experimental discovery of the VB mode, clearly described by de Haas et al. [5], had been overlooked in the literature. Recently, an experimental determination of the phase diagram was reported [19], and these results were considered for the present study.

This paper is organized as follows. In Sec. II, the vesicle model is presented. The small deformation theory and its associated dynamical equations appear in Sec. III. Section IV contains the main results and discussion. Section V presents a brief summary of the main outcomes and some comments.

\section{VESICLE MODEL}

\section{A. Membrane structure and bending force}

At room temperature, the membrane is in a liquid state. The energy required to expand or to compress a portion of the membrane is very high, i.e., the membrane compressibility modulus is $\sim 100 \mathrm{mN} / \mathrm{m}[1]$. Therefore, the membrane is considered as a two-dimensional incompressible Newtonian fluid and this implies local and global conservation of the membrane surface area. This conservation is not observed for other deformable particles, such as capsules or droplets. RBCs share this surface conservation property. The difference between vesicles, drops, and capsules has recently been discussed $[14,17]$. Moreover, at osmotic equilibrium there is no net flow across the membrane, so the enclosed volume is conserved.

Energy is stored in the membrane bending modes. The associated energy is given by [24]

$$
E=\frac{\kappa}{2} \int_{\partial \Omega}(2 H)^{2} d A+\int_{\partial \Omega} \zeta d A .
$$

The force is obtained by the derivative of the energy with respect to the membrane shape [25],

$$
\mathbf{F}=\left(\kappa\left[2 H\left(2 H^{2}-2 K\right)+2 \Delta_{l b} H\right]-2 \zeta H\right) \mathbf{n}+(I-\mathbf{n} \otimes \mathbf{n}) \cdot \nabla \zeta,
$$

where $\kappa$ is the membrane rigidity $\left(\sim 10^{-19} \mathrm{~J}\right)$, $H=\left(1 / R_{1}+1 / R_{2}\right) / 2$ and $K=1 /\left(R_{1} R_{2}\right)$ are the mean and the Gaussian curvatures, respectively $\left(R_{1}\right.$ and $R_{2}$ are the two principal radii of curvature), $\Delta_{l b}$ is the Laplace-Beltrami (surface) operator, $\zeta$ is a Lagrange multiplier that enforces local membrane surface conservation, $I$ is the identity tensor, and $\mathbf{n}$ is the outward unit normal vector.

\section{B. Fluid equations and their dimensionless form}

In this section, the model is presented in its dimensionless form in order to identify the three dimensionless parameters. When the vesicle is subjected to linear shear flow,

$$
v_{x}^{0}=\gamma y, \quad v_{y}^{0}=v_{z}^{0}=0,
$$

where $\gamma$ is the shear rate.

The following dimensionless variables are introduced for space, time, fluid velocity, and pressure fields:

$$
\mathbf{r}^{*}=\mathbf{r} / R_{0}, \quad t^{*}=t / t_{s}, \quad \mathbf{v}^{*}=\mathbf{v} / U, \quad p^{*}=p R_{0} / \eta U,
$$

where $t_{s}$ and $U$ are characteristic time and velocity scales associated with the flow. The quantity $t_{s}=R_{0} / U=1 / \gamma$ is 
fixed, since this is the only time scale associated with the flow. We take the viscosity units to be that of the ambient fluid, $\eta_{\text {ext }}$.

For most available experimental data, the Reynolds number is small (typically $10^{-2}-10^{-3}$ ), and thus inertia can be neglected. The Stokes equations result, whose dimensionless form inside and outside the vesicle, are

$$
\left\{\begin{array}{c}
\lambda \nabla^{* 2} \mathbf{v}_{\text {int }}^{*}-\nabla^{*} p_{\text {int }}^{*}=0, \\
\nabla \cdot \mathbf{v}_{\text {int }}^{*}=0
\end{array}\right.
$$

and

$$
\left\{\begin{array}{c}
\nabla^{* 2} \mathbf{v}_{\mathrm{ext}}^{*}-\nabla^{*} p_{\mathrm{ext}}^{*}=0 \\
\nabla^{*} \cdot \mathbf{v}_{\mathrm{ext}}^{*}=0
\end{array}\right.
$$

where $\lambda$ is the viscosity contrast [see Eq. (2)].

At the membrane, the bending force must balance the change in the hydrodynamic stress,

$$
\left(\sigma_{\mathrm{ext}}^{*}-\sigma_{\mathrm{int}}^{*}\right) \mathbf{n}+\mathbf{F}^{*}=0,
$$

where $\sigma^{*}$ is the dimensionless stress tensor given in each fluid domain by $\sigma_{\text {ext }}^{*}=-p_{\text {ext }}^{*} I+\left[\nabla^{*} v_{\text {ext }}^{*}+\left(\nabla^{*} v_{\text {ext }}^{*}\right)^{T}\right]$ and $\sigma_{\text {int }}^{*}=-p_{\text {int }}^{*} I+\lambda\left[\nabla^{*} v_{\text {int }}^{*}+\left(\nabla^{*} v_{\text {int }}^{*}\right)^{T}\right]$ (the superscript " $T$ " signifies the transpose of the matrix). The dimensionless membrane force takes the form

$$
\begin{aligned}
\mathbf{F}^{*}= & \left\{\mathrm{Ca}^{-1}\left[2 H^{*}\left(2 H^{* 2}-2 K^{*}\right)+2 \Delta_{l b}^{*} H^{*}\right]-2 \zeta^{*} H^{*}\right\} \mathbf{n} \\
& +(I-\mathbf{n} \otimes \mathbf{n}) \cdot \nabla^{*} \zeta^{*},
\end{aligned}
$$

where $\mathrm{Ca}$ is the capillary number given by Eq. (3), and $\zeta^{*}=\zeta /\left(\eta \gamma R_{0}\right)$ is the dimensionless Lagrange multiplier. The additional equations that are needed to determine this unknown are obtained from the local membrane incompressibility condition, which can be expressed as the constraint on the velocity field at the surface of the vesicle,

$$
\left(\delta_{i j}-n_{i} n_{j}\right) \partial_{i} v_{j}^{*}=0 .
$$

The third dimensionless parameter is the excess area relative to a sphere, $\Delta$, that quantifies by how much the vesicle is deflated relative to a sphere.

At the membrane the fluid velocity is continuous, which implies

$$
\mathbf{v}_{\mathrm{ext}}^{*}=\mathbf{v}_{\text {int }}^{*} \cdot
$$

If the membrane does not allow for a net flow, then the membrane velocity $\mathbf{v}_{m}^{*}$ is equal to that of the adjacent fluid,

$$
\mathbf{v}_{m}^{*}=\mathbf{v}_{\text {ext }}^{*}=\mathbf{v}_{\text {int }}^{*} \text {. }
$$

Finally, at large distances from the vesicle, the flow approaches the imposed one (formally, the induced flow due to the presence of the vesicle vanishes at infinite distances from the vesicle)

$$
\mathbf{v}_{\mathrm{ext}}^{*}(r \rightarrow \infty)=\mathbf{v}^{* 0} .
$$

Equations (9)-(16) constitute a closed set for the study of the dynamics of a vesicle in a shear flow. The solutions for the velocity and pressure field are obtained following Lamb's procedure [26], and their expressions together with other de- tails can be found in Ref. [23]. From now on, the superscript “*” will be omitted, and only dimensionless quantities will be considered.

\section{SMALL DEFORMATION THEORY}

In the small deformation theories, the dynamics and deformation of a particle, due to an external applied flow, are obtained by performing an expansion about the simple geometry, namely, a sphere (vesicles $[5,6,14]$, droplets $[27,28]$, and capsules $[29,30])$.

\section{A. Vesicle shape}

The surface of the vesicle, in spherical coordinates $(\theta$ $\in[0, \pi]$ and $\phi \in[0,2 \pi])$, is determined by the vector position $[14]$

$$
\mathbf{R}(\theta, \phi)=[1+\epsilon f(\theta, \phi)] \mathbf{e}_{\mathrm{r}},
$$

where $\epsilon$ is a small parameter related to the vesicle excess area $(\epsilon=\sqrt{\Delta})$, and which is used as an expansion parameter for the deviation from a sphere $[15,23]$. The function, $f$, is decomposed into the spherical harmonics, $Y_{2}^{m}(\theta, \phi)$ as

$$
f(\theta, \phi)=\sum_{m=-2}^{2} F_{2, m} Y_{2}^{m}=F_{2,-2} Y_{2}^{-2}+F_{2,0} Y_{2}^{0}+F_{2,2} Y_{2}^{2},
$$

where $F_{2, m}$ is a time-dependent amplitude of the corresponding mode, the evolution equations of which will be analyzed. The amplitude associated with $Y_{0}^{m}$ can be expressed in terms of the other amplitudes, via the vesicle volume conservation constraint $[6,14,23]$, and the mode $Y_{1}^{m}$ is omitted, since we are not interested in translation of the vesicle. Since we consider a vesicle under simple linear shear flow, only the spherical harmonics of order $n=2$ survive [14]. This is sufficient to capture the basic features of dynamics. Finally, in Eq. (18), the harmonics $Y_{2}^{ \pm 1}$ have been omitted since the dynamics are only analyzed in the plane of the shear flow. Thus, $Y_{2}^{ \pm 1}=0$ at $\theta=\pi / 2$; the shear plane is $x-y$.

\section{B. Shape evolution equations}

The time evolution of the shape is given by the dynamics of the $F_{i, j}$ modes (see Ref. [23]). This work follows the socalled post-expansion theory, which keeps the leading terms in the full evolution equations in a consistent manner [23]. The equality [14]

$$
F_{2, \pm 2}=\mathcal{R} e^{\mp 2 i \Psi}
$$

is set and, with this definition in the TT regime, $\Psi$ coincides with the orientation angle of the vesicle main axis, and $\mathcal{R}$ is the amplitude of the vesicle deformation. For ease of comparison, instead of $\mathcal{R}$, the variable $\Theta$ [16] can be used, as defined by $2 \mathcal{R}=\cos \Theta$ with $0 \leq \Theta<\pi$. Since in Eq. (17), the deformation amplitude is multiplied by $\epsilon=\sqrt{\Delta}$, it is more convenient to study the full amplitude, $R=\frac{\sqrt{\Delta}}{2} \cos \Theta$. Following the post-expansion theory [23], the relevant leading order expansion about a sphere is obtained 


$$
\begin{aligned}
T \frac{\partial \Theta}{\partial t}= & -S \sin \Theta \sin 2 \Psi+\cos 3 \Theta \\
& +\Lambda_{1} S \sin (2 \Psi)(\cos 4 \Theta+\cos 2 \Theta) \\
& T \frac{\partial \Psi}{\partial t}=\frac{S}{2}\left[\frac{\cos 2 \Psi}{\cos \Theta}-\Lambda\right]
\end{aligned}
$$

with

$$
\begin{aligned}
T & =\frac{7 \sqrt{10 \pi}}{720} \frac{(23 \lambda+32) \mathrm{Ca}}{\sqrt{\Delta}}, \\
\Lambda_{1} & =\frac{1}{28} \sqrt{\frac{10}{\pi}} \frac{49 \lambda+136}{23 \lambda+32} \sqrt{\Delta},
\end{aligned}
$$

and $S$ and $\Lambda$ are given in Eq. (4). The above coefficients are related to the three independent parameters $\Delta, \lambda$, and $\mathrm{Ca}$. The dimensionless parameter $T$ can be absorbed in a redefinition of time so that three independent parameters remain: $S, \Lambda$, and $\Lambda_{1}$. In the following discussion, either one of the two sets, $(\Delta, \lambda, \mathrm{Ca})$ or $\left(S, \Lambda, \Lambda_{1}\right)$, will be used. The first set is more natural, since it has a simple physical interpretation, but the second set allows for comparison with Ref. [16]. Note that Eqs. (20a) and (20b) are symmetric under the transformations,

$$
\left\{\begin{array} { c } 
{ \Theta \rightarrow \Theta } \\
{ \Psi \rightarrow \Psi + \pi }
\end{array} \text { and } \left\{\begin{array}{c}
\Theta \rightarrow \pi-\Theta \\
\Psi \rightarrow \Psi+\frac{\pi}{2} .
\end{array}\right.\right.
$$

This is a useful observation since the numerical solutions (by means of the Newton method) for the fixed points of Eqs. (20a) and (20b) typically lead (for a given set of parameters) to several branches, which may be unintuitive, yet simply, related to each other via the above symmetry relations.

The first term on the right-hand side of Eq. (20a) is on order $1 / \epsilon^{2}$ (recall that $\epsilon=\sqrt{\Delta}$ ), the second one is on order 1 , and the final one is on order $1 / \epsilon$. The first contribution $\left(1 / \epsilon^{2}\right)$ arises in the leading theory [14], the second is the term added in [16], and the consistent calculation [23] includes the third term. The consistent theory has been discussed at length in [23]. Actually, the parameters $S$ and $\Lambda$ represent the asymptotic behavior of characteristic values of $\mathrm{Ca}$ and $\lambda$ for small values of $\Delta$. If it is assumed that $\mathrm{Ca}$ scales as (i.e., it is on the same order) $\Delta$ and $\lambda$ scales as $\Delta^{-1 / 2}$, then the last term in Eq. (20a) can be neglected for sufficiently small $\Delta$ and the dynamical properties of the vesicle become dependent only on two parameters, $\Lambda$ and $S$ $(T=\Lambda S / \gamma)$. In that case, the two theories [16,23] agree. However, if $\mathrm{Ca}$ remains on order $O(1)$, then the last term in Eq. (20a) plays an important role, especially in the VB and TB regimes. The goal of the present study is to provide a detailed analysis of the consequences of the consistent theory [23].

Equation (20b) provides the evolution of the vesicle inclination angle, $\Psi(-\pi \leq \Psi \leq \pi)$. For a given set of solutions, $\{\Theta(t), \Psi(t)\}$ (recall that $R$ is related to $\Theta) F_{2,2}$ [see Eq. (19)] and $F_{2,-2}$ are obtained. The quantity $F_{2,0}$ is obtained via the area conservation constraint (for details see [23]), which re- flects the fact that the deformation amplitudes must comply with the available excess area. This condition is manifested as $2 F_{2,0}^{2}+4\left|F_{2,2}\right|^{2}=1$, which fully determines the vesicle shape configuration.

\section{RESULTS}

The following results are obtained by numerically solving Eqs. (20a) and (20b), which are nonlinear ordinary differential equations of first order, using Maple.

\section{A. Phase diagram}

\section{General consideration}

First, the phase diagram corresponding to the various regimes will be considered. Three regimes were identified under shear flow (see Fig. 3): (i) tank-treading (TT) (blue area), (ii) vacillating-breathing (VB) (violet area), and tumbling (TB) (red area). The phase diagram was derived using Eqs. (20a) and (20b). The steady TT to unsteady VB transition border, in the phase diagram, was observed when at least one eigenvalue, $\omega$, of the stability matrix of the set of fixed points corresponding to TT had a real part which became positive (perturbations of the fixed point as $\sim e^{\omega t}$ ). The occurrence of VB mode corresponds to a Hopf bifurcation: the real part of $\omega$ vanishes, while its imaginary part is finite.

The boundaries between the two unsteady regimes, TB and VB, was obtained numerically. TB is the continuation of the VB mode when $\Psi$ reaches $\pm \pi / 4$. In the low deformation regime $(\mathrm{Ca}<1)$, the transition from TT to TB is direct (i.e., it is not preceded by a VB mode; see Fig. 3) and it occurs via a saddle-node bifurcation, as discussed in Ref. [7]. In the higher deformation regime $(\mathrm{Ca}>1)$, TB is preceded by a VB regime upon increasing $\lambda$. Note that a small $\mathrm{Ca}$ implies that the vesicle response is fast in comparison to the shearing time; the vesicle adapts instantaneously to the shape imposed by the flow. A large $\mathrm{Ca}$ implies that the vesicle response is slow in comparison to the shearing time, and therefore, the vesicle will exhibit ample shape variation. Figure 4 shows the behavior of the orientation angle of the long axis of the vesicle, $\Psi$, as a function of time in the three regimes (from left to right TT, VB, and TB). The VB and TB modes are also shown in a movie [21]. The movie clearly displays the large breathing of the vesicle in the VB regime. A more subtle effect is that, in the TB regime, the vesicle axes also undergo an oscillation. A close inspection of the TB regime shows that the two main axes exhibit an oscillation in time.

The phase diagram (Fig. 3) has been discussed by three groups $[16,22,23]$, and there is a consensus on its qualitative shape. However, further investigations are warranted to reveal how the three control parameters affect the phase and physical quantities, such as the period and amplitude of oscillations. This work clarifies these points through a careful analysis of the dynamics, which allow for several important disagreements between the three groups to be identified.

\section{Significant dependence of the boundaries of different regimes in parameter space}

First, the evolution of the phase diagram (Fig. 3) with $\Delta$ is investigated. A typical result is shown in Fig. 5. The phase 


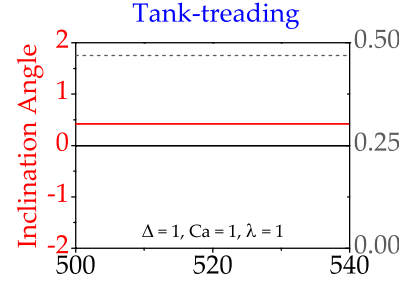

(a)

Time

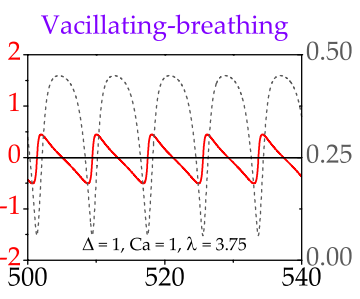

(b)
Time

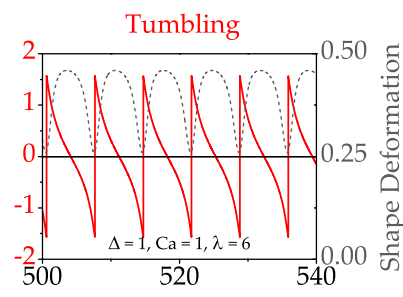

(c)

Time

FIG. 4. (Color online) Time evolution (time in unit of inverse shear rate) of the vesicle inclination (in red full line) angle and its shape deformation (in dashed line) for the three different dynamical regimes: (a) tank-treading, (b) vacillating-breathing, and (c) tumbling. A precise definition of the shape deformation is given in subsection shape evolution equations (below). In the TT regime, only the permanent regime where the angle is constant in time is shown.

diagram can be characterized by three quantities: the critical viscosity contrast $\left(\lambda_{C}\right)$, the critical capillary number $\left(\mathrm{Ca}_{C}\right)$, and the width of the VB domain $(\Delta \lambda)$ at high enough $\mathrm{Ca}$, as shown in Fig. 5. These three quantities are presented in Fig. 6 as a function of $\Delta$. The increase of $\lambda_{C}$ with decreasing $\Delta$ has already been reported $[3,8,13]$. However, the dependence of the width of the VB mode as a function of $\Delta$ has not been reported.

As discussed above, it was claimed in Ref. [16] that two independent control parameters, $S$ and $\Lambda$, are sufficient to describe the vesicle dynamics. In order to test this idea, the phase diagram in the $S-\Lambda$ plane was investigated. The results are shown in Fig. 7. According to Ref. [16], the phase diagram is universal in that plane (black line in Fig. 7). Clearly, this assumption is not valid, as shown in the same figure. By varying the excess area, a significant variation in the boundaries separating the TT-VB and VB-TB regime was observed. In fact, the additional term $\left(\Lambda_{1}\right)$ appearing on the right-hand side of Eq. (20a) strongly influences the phase diagram border location, making it quite sensitive to the ex-

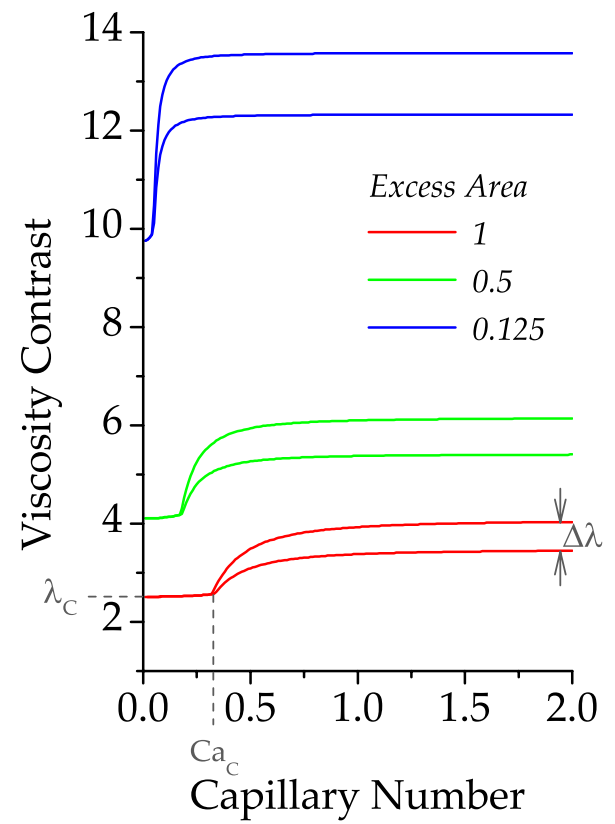

FIG. 5. (Color online) Evolution of the phase diagram borders as a function of the vesicle excess area. $\Delta=1,0.5,0.125$, from bottom to top curves. cess area parameter. It is only when $\mathrm{Ca}$ (or $S$ ) is sufficiently small that the two theories coincide. Recent experiments [19] have reported a phase diagram in the $S-\Lambda$ plane by averaging out all values of excess area. In light of the results presented here, this is not appropriate. Different values of $\Delta$ imply different locations of the bifurcation boundaries, which may be used to interpret future experiments.

The values of $\Delta$ used above are consistent with most of the available experimental data, which have been in the range $[5,11,13] \Delta=0.5-1.5$. The third parameter $\Delta$ will be seen to influence the amplitude of the VB mode even stronger (when $S$ and $\Lambda$ are fixed).

\section{B. Tank-treading: Dependence of the steady angle on shear rate}

In 3D numerical simulations [4], it was reported that the inclination angle in the tank-treading regime does not depend on the shear rate (or more precisely on $\mathrm{Ca}$ ). We find here that this does not always hold. Figure 8 shows, for a given set of

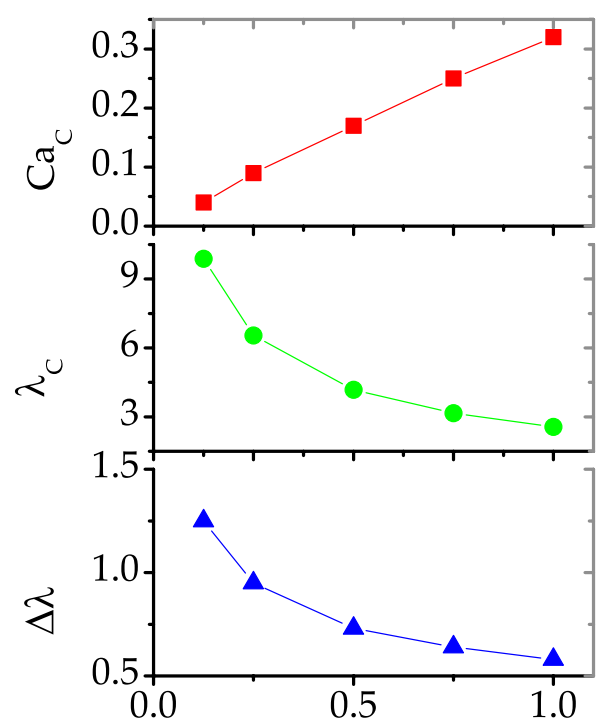

\section{Excess Area $\Delta$}

FIG. 6. (Color online) The behavior of $\mathrm{Ca}_{c}, \lambda_{c}$, and the width of the VB mode at high enough $\mathrm{Ca}$ as functions of the excess area, $\Delta$. We see that the VB width significantly shrinks upon increasing $\Delta$. 


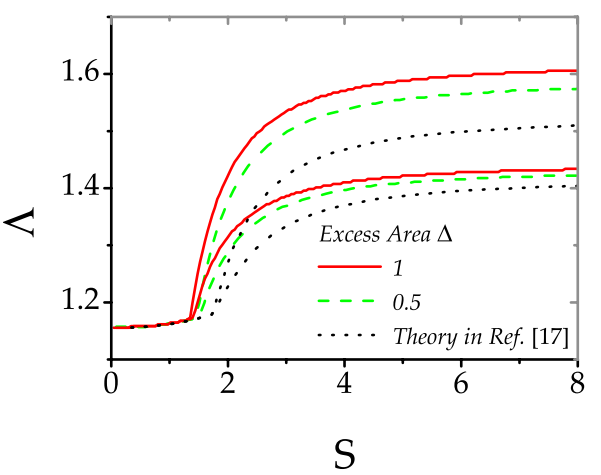

FIG. 7. (Color online) Comparison between the two phase diagrams obtained by the present theory (in color full and dashed lines) and the one reported in Ref. [16] (dotted black line) for three different values of the excess area. In both cases, the phase diagram is drawn in the $S-\Lambda$ plane (as in Ref. [16]).

viscosity ratios, the variation in the steady inclination angle when varying $\mathrm{Ca}$. At low shear rates $(\mathrm{Ca} \ll 1)$, the steady inclination angle decreases with increasing $\mathrm{Ca}$. This decrease may be significant (see Fig. 8 for $\lambda=2$ ) where it can attain a factor of about 2 . This behavior was briefly commented upon in the experiments of Ref. [5]. A systematic experimental analysis of this phenomenon is lacking. The current physical interpretation relies on a decomposition of simple shear flow into an elongation and a rotational component. The elongational component "stretches" the vesicle along the TT direction. At low $\mathrm{Ca}$, the vesicle main axis is not yet completely elongated. As $\mathrm{Ca}$ increases, the vesicle continues to elongate, and the effect of the torque (due to the rotational component) is enhanced, causing a further inclination of the vesicle toward the flow direction. When $\mathrm{Ca}$ is large enough, the vesicle elongation saturates, and consequently the angle reaches a plateau. Note that the plateau is reached earlier for a low viscosity contrast, because for low $\lambda$, the TT inclination angle more closely approaches the direction of maximum elongation, $\pi / 4$. Thus, the elongation is more efficient, and maximum elongation can be attained at a lower shear

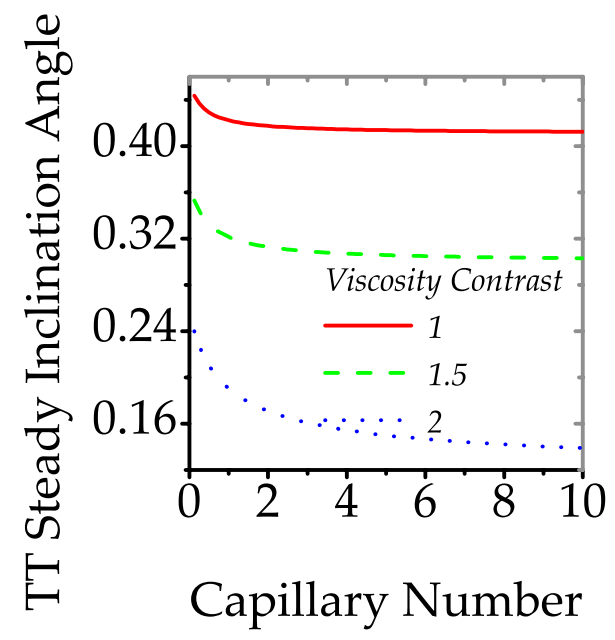

FIG. 8. (Color online) The steady inclination angle, in radians, of a vesicle performing TT versus Ca for different values of $\Delta$. Here, $\Delta=1$.

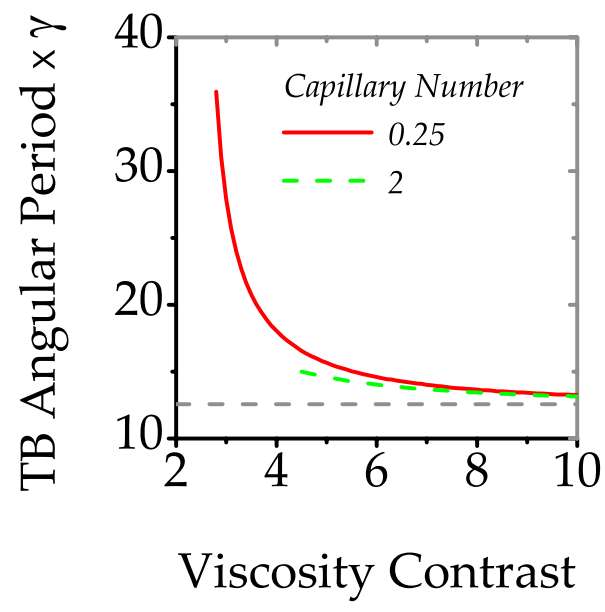

FIG. 9. (Color online) The period of vesicle tumbling (rescaled by $\gamma$ ), versus the viscosity contrast for different values of the capillary number. The measurements begin with the viscosity contrast corresponding to the threshold of the transition to tumbling regime (see Fig. 3). The dashed gray horizontal line corresponds to the period of a rotating rigid body (Ref. [31]). Here, $\Delta=1$.

rate. Finally, the fact that, for a given $\mathrm{Ca}$, the angle decreases upon increasing $\lambda$, is a classical result [3,7-9].

The result for the variation in the TT angle with $\mathrm{Ca}$, shown in Fig. 8, was not observed in the full simulation by Kraus et al. [4] for the following reasons. That work was limited to $\lambda=1$, and $\mathrm{Ca} \geq 1$. As can be seen in Fig. 8 (red line), the plateau is reached for $\lambda=1$ slightly above $\mathrm{Ca}=1$, which could not have easily been reproduced in the simulations of Kraus et al. [4]. As shown here, if one takes $\lambda=2$ (blue line in Fig. 8), the plateau is reached at $\mathrm{Ca} \simeq 10$. The range $\mathrm{Ca}=1-10$ is experimentally accessible $[11,13]$, and we hope that these results will inspire systematic investigations in future experiments.

\section{Tumbling}

\section{Behavior of the TB period with $\lambda$}

At large enough $\mathrm{Ca}$, the TB period decreases slowly with increasing $\lambda$ (Fig. 9). It is only in the small Ca regime (i.e., when there is a direct bifurcation from TT to TB, see Fig. 5) that the period varies abruptly with $\lambda$ (with a period approaching infinity at the transition point due to the Landau critical slowing down). To our knowledge, the behavior of the TB angular period with $\lambda$ has not yet been reported. The dashed gray line in Fig. 9 corresponds to the period of a quasispherical rotating rigid body with a frequency $\Omega=\gamma / 2$ [31]. Note that all TB angular period curves tend to this value at higher $\lambda$. The decrease in the period with $\lambda$ is interpreted as follows. At small $\lambda$, the vesicle deformation is high. During $\mathrm{TB}$, the long and short axes oscillate (maximal stretching occurs at $\pi / 4$ and maximal compression at $\pi=-\pi / 4)$ and, due to accumulation of vesicle stretching during TB motion in the $y>0$, the vesicle approaches the horizontal geometry in a stretched state, and will spend more time there before entering the $y<0$. When $\lambda$ is larger, the stretching is decreased due to the flow inside the vesicle. 
This enhances the delay between the shearing time and the response of the vesicle. The vesicle, being less stretched, will thus spend less time in the horizontal direction.

\section{Behavior of the $\mathrm{TB}$ period with $\mathrm{Ca}$}

We have investigated the TB period as a function of $\mathrm{Ca}$, for different values of $\lambda$ (the behavior of the period is not shown here due to the slight sensitivity to the parameters). In the low deformation regime $(\mathrm{Ca} \ll 1)$, we find a weak decrease in the TB period upon increasing $\mathrm{Ca}$. The decrease is typically on the order of $6 \%$ to $11 \%$. In the larger deformation regime, more precisely when $\mathrm{Ca} \sim 2$, the period nearly ceases to depend on $\mathrm{Ca}$ for the range of $\lambda$ values explored (from 1 to 10). The fact that the oscillation period is quite insensitive to $\mathrm{Ca}$ may be explained as follows. At high enough $\mathrm{Ca}$ (say beyond 2), the vesicle stretching will reach a maximal value, such that the vesicle shape does not evolve further. The vesicle has nearly the same shape (given the fact that $\lambda$ and $\Delta$ are fixed), and nothing causes the period (normalized by the shear rate) to change.

We would like to draw attention on a contradiction between the present work and that of Noguchi and Gompper [22], who have also reported on the behavior of the TB period as a function of $\mathrm{Ca}$ (for their frequency, see Fig. 2 therein). They observed an increase in the period with $\mathrm{Ca}$, while the opposite behavior was seen here. The most significant point is that, the period they observed strongly depended on $\mathrm{Ca}$ (their period varies by about a factor 4 when $\mathrm{Ca}$ is varied by a similar factor). In contrast, we find variations in few \%. The insensitivity of the TB period to $\mathrm{Ca}$ has also been observed numerically using a boundary integral method in three dimensions (to be reported elsewhere [32]). Noguchi and Gompper [22] used a dissipative particle numerical simulation technique with a membrane viscosity (not included in our study). The effect of the membrane viscosity has been included in the analytical theory [16], but it simply results in a renormalization of the bulk viscosities, a fact which cannot change our results regarding the dependence of the period on Ca. Noguchi and Gompper [22] additionally used a phenomenological picture based on the KS theory that seemed to support their finding. Neither the present study nor the full 3D numerical simulation [32] agree with Noguchi and Gompper's result. Our full numerical boundary integral simulations show a marked contrast with the KS theory [32]. The strong dependence on Ca observed by Noguchi and Gompper [22] in the phenomenological study (using KS theory) might be symptomatic of the breakdown of the KS theory. However, there is currently no explanation for why their numerical simulations provide quite different results than ours (both from our analytical and numerical data).

\section{Vacillating-breathing}

\section{Vacillating-breathing period}

Figure 10 shows the VB angular period versus $\mathrm{Ca}$, for different values of $\lambda$. For a given $\mathrm{Ca}$, the $\mathrm{VB}$ period decreases with increasing $\lambda$. This can be rationalized by noting that increasing $\lambda$ reduces the vesicle deformation and makes

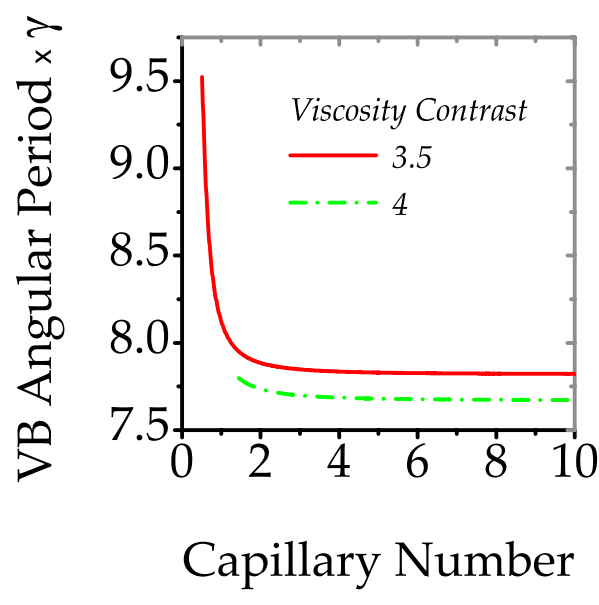

FIG. 10. (Color online) The period of vacillating-breathing (rescaled by $\gamma$ ), versus the capillary number for different values of the viscosity contrast. Here, $\Delta=1$.

the VB response stiffer and stiffer, which leads to a faster motion (smaller period).

For a given $\lambda$, it is only in the vicinity of the transition from TB to the VB mode (compare Fig. 5 for $\Delta=1$ to the data in Fig. 11(b) in order to locate the various regimes) that the period undergoes an abrupt drop (a consequence of the critical slowing down). By increasing $\mathrm{Ca}$ beyond a typical value of about 2 , the period reaches a plateau, as it did in the TB regime. The observation that the period is insensitive to $\mathrm{Ca}$ (for $\mathrm{Ca}$ on order of, or greater than, 2) has been confirmed by the full three-dimensional simulations based on the boundary integral formulation, to be reported elsewhere [32].

\section{Vacillating-breathing amplitude: Nature of the bifurcation from $T T$ to $V B$}

The (complex) amplitude of the VB mode is now investigated, which is sensitive to the three dimensionless parameters. The VB angular amplitude, $\Delta \Psi$ (defined as the absolute value of the difference between the maximum and the minimum of $\Psi(t))$, increases with $\lambda$, as is depicted in Fig. 11(a). It tends to approach $\pi / 2$ when $\lambda$ is close to the TB boundary. When $\lambda$ is close to the TT boundary, both the minimum and the maximum of $\Psi(t)$ tend to zero.

The fact that the amplitude approaches zero at TT-VB boundary in a continuous manner was observed for all the parameter values explored so far. Therefore, the bifurcation from TT to VB is supercritical (in contrast to a subcritical bifurcation; a dynamical analog of a first order transition). This feature is further investigated by plotting the amplitude as a function of $\lambda$. The typical behavior is shown in Fig. 11(a). The amplitude is well fitted with a square root law $\left(\Delta \Psi \sim \sqrt{\lambda-\lambda_{C}}\right)$ in the vicinity of the bifurcation point, which is a prototypical result for a supercritical (or pitchfork) bifurcation.

Note that absolute value of the minimal angle is different from the maximal one, $\left|\Psi_{\min }\right| \neq\left|\Psi_{\max }\right|$ with $\left|\Psi_{\min }\right|>\left|\Psi_{\max }\right|$. In the VB mode, the longest axis of the vesicle oscillates around a small negative angle (in contrast to RBCs that oscillate around a positive angle [33]). This means that the 


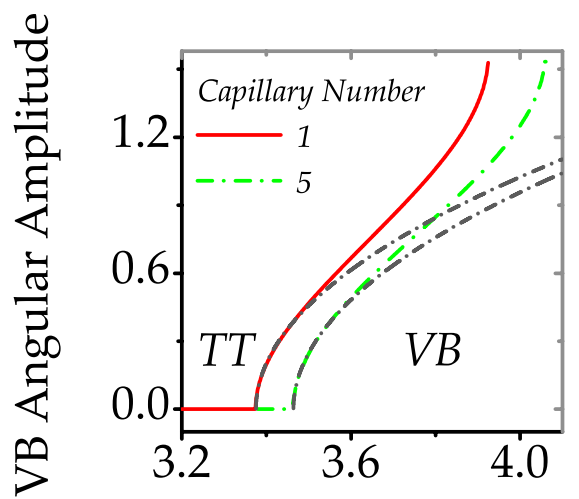

(a) Viscosity Contrast

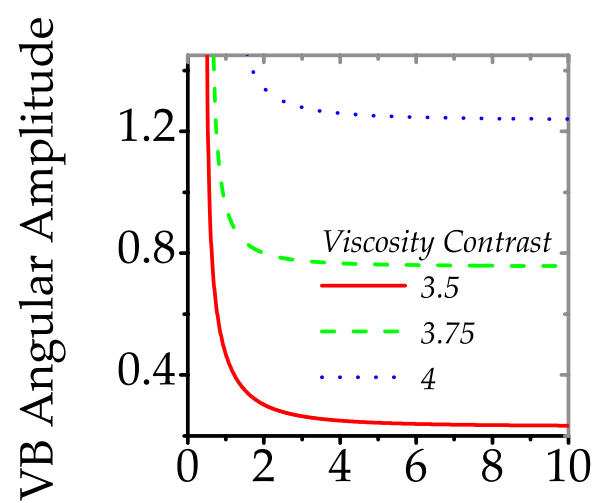

\section{(b) Capillary Number}

FIG. 11. (Color online) The angular amplitude, in radians, of vacillating-breathing mode, versus the viscosity contrast for different values of the capillary number are plotted. The dashed-dotted lines are fits of the angular amplitude with a square root law $\left(\sim \sqrt{\lambda-\lambda_{C}}\right)(\mathrm{a})$; and versus the capillary number for different values of the viscosity contrast (b). Here, $\Delta=1$

oscillation takes place about an angle that is very close to that of a TT vesicle before the TT-VB transition occurs. Figure 11(b) shows the VB angular amplitude versus Ca for different values of $\lambda$. At smaller values of $\mathrm{Ca}$, the amplitude decreases with increasing $\mathrm{Ca}$, until it reaches a plateau, at higher values. The same behavior was also reported in [22].

\section{Vacillating-breathing amplitude: Strong dependence on the three control parameters}

Figure 12 represents the variation in the angular and the deformation amplitudes with the excess area, $\Delta$, in the VB regime. The values of the parameters $S$ and $\Lambda$ used in Fig. 12 were chosen to select the VB regime. Subsequently, $S$ and $\Lambda$ were fixed and $\Delta$ was varied from 0.125 to 1 (a typical range in experiments [5,11-13,19]). The angular amplitude decreases with $\Delta$, while the deformation amplitude increases. At higher $\Delta$ (more deflated vesicles), ample breathing is caused, which can be measured by $R$. Due to this ample breathing, the main axis of the vesicle remains quite close to the horizontal axis, which implies that the amplitude of vacillation (measured by $\Psi$ ) decreases.

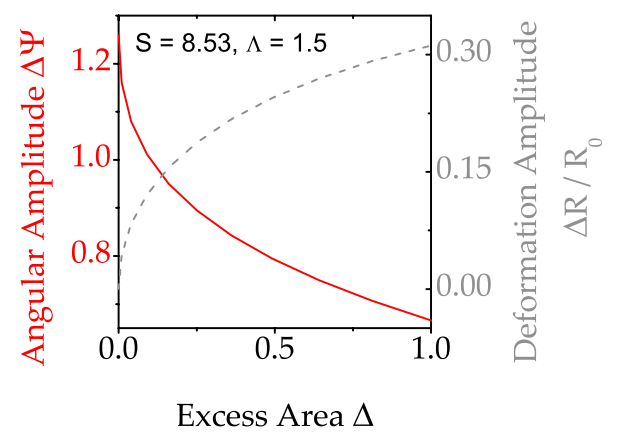

FIG. 12. (Color online) Variations in both the angular amplitude in radians (the left axis) and the deformation, rescaled by $R_{0}$ (the right axis) as a function of the excess area, for a given set of $S$ and $\Lambda$ parameters.

An important fact is the strong variation in both $R$ and $\Psi$ with $\Delta$, for a fixed set of $S$ and $\Lambda$. These results confirm the importance of the excess area as a third control parameter. Unfortunately, the recent experimental study [19] (where the authors neglected the third parameter) did not investigate this quantity, which is essential to verifying the theory.

\section{Vacillating-breathing limit-cycles}

Figure 13 shows the limit-cycles of a vesicle performing VB dynamics for different sets of parameters. A point belonging to a limit-cycle [e.g., the point A in Fig. 13(a)] represents the instantaneous vesicle inclination angle and the corresponding deformation.

In Fig. 13(a), a typical limit-cycle for the VB mode and its evolution with $\Delta$ are shown, while $S$ and $\Lambda$ are fixed, as in Fig. 12. Increasing the excess area induces a shift in the limit-cycle toward higher deformation regions, and to smaller amplitude angular oscillations.

It must be noted that, when varying $\mathrm{Ca}$, for the range observed in Fig. 13(b) and while $\lambda$ and $\Delta$ are fixed, the configuration of the limit-cycle does not exhibit a dramatic change. Only small variations in the deformation and the angular amplitudes were observed.

Figure 13(c) shows the evolution of the limit-cycle when varying $\lambda$. Increasing $\lambda$ causes an increase in the deformation and the angular amplitudes. The same information is represented in Fig. 13(d), and in the $\Theta-\Psi$ Atlas.

\section{CONCLUSION}

Using the dynamical equations, (20a) and (20b), derived in Ref. [23], a systematic physical analysis of vesicle dynamics under a linear shear flow was performed.

It was observed that the boundaries of the phase diagram corresponding to the TT, VB, and $\mathrm{TB}$ regimes are sensitive to the three dimensionless control parameters, and not only the two reported in Ref. [16] ( $S$ and $\Lambda$ ). It has been shown that the amplitudes of deformation and orientation in the VB strongly depend on the third parameter $\Delta$ (by fixing $S$ and $\Lambda)$. It has also been shown that the vesicle inclination angle in the TT regime significantly depends on shear rate in the parameter range that is accessible to experiments. Finally, it 
(a)
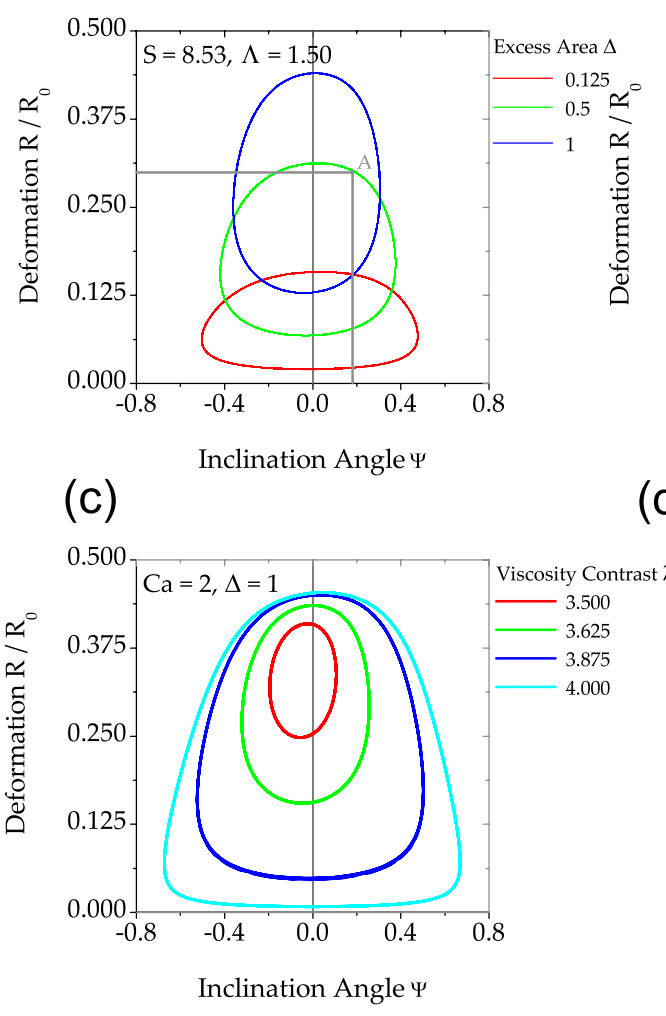

(b)
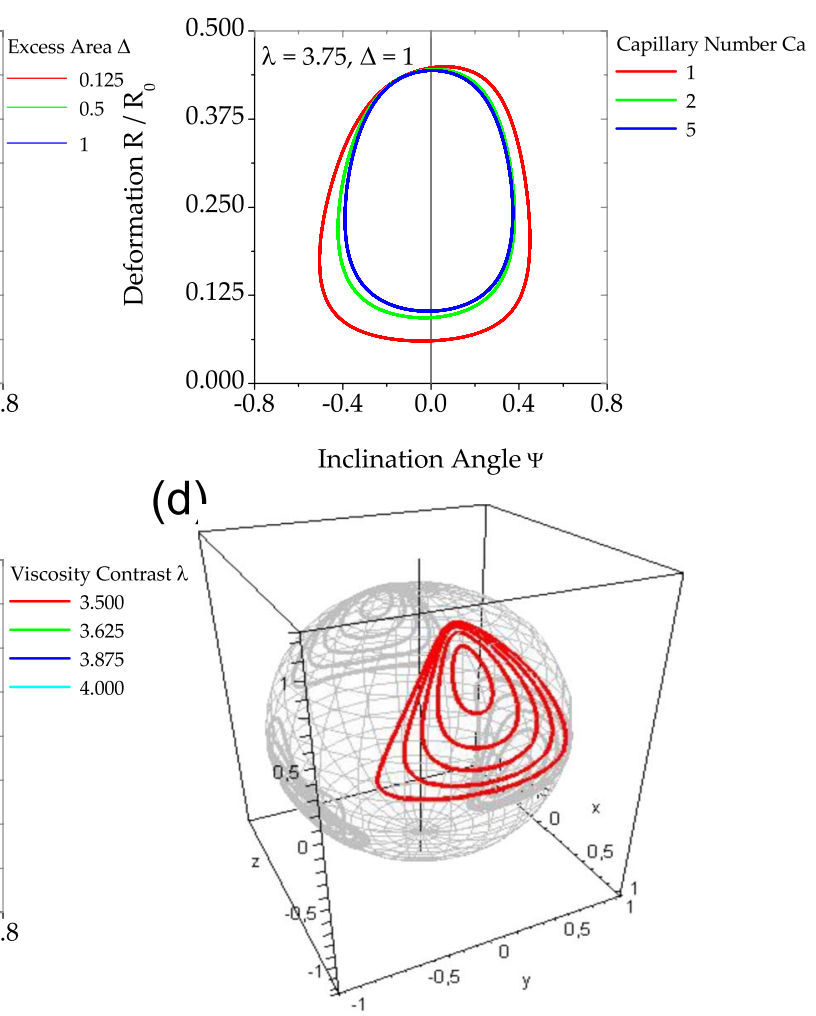

FIG. 13. (Color online) Plot of the evolution of the limit-cycle configuration (in the $R-\Psi$ plane) of a vacillating-breathing vesicle, under shear flow, when varying either the excess area (a), the capillary number (b), or the viscosity contrast (c). Plot of the evolution of the limit-cycle configuration in the Atlas $\Theta-\Psi$ when varying the viscosity contrast.

was reported that the period of oscillation in the VB and TB regimes is quite insensitive to the shear rate. This result is in contradiction with previously reported results based on phenomenological equations and on dissipative particle dynamics [22].

Recent experiments [19] have reported the phase diagram (such as Fig. 7) and have concluded, by reference to the work of Lebedev et al. [16], that only the two parameters $S$ and $\Lambda$ are relevant. In the light of the present study, this conclusion is inappropriate. A careful analysis should be performed by selecting vesicles with different excess areas. In addition, experimental investigations of several other relevant physical quantities are lacking (such as the amplitude of the VB mode, as in Fig. 11, and the behavior of the limitcycle, as in Fig. 13), where a strong variation with the third parameter is expected. We hope that this work will inspire such experiments.

\section{ACKNOWLEDGMENTS}

Financial support from CNES, ANR (MOSICOB), and EGIDE PAI Volubilis (Grant No. MA/06/144) is acknowledged. B.K. would like to thank G. Danker and T. Podgorski for fruitful and helpful discussions and CNRST for the grant (Grant No. b4/015).
[1] R. Lipowsky and E. Sackmann, Structure and Dynamics of Membranes, from Cells to Vesicles (North-Holland, Amsterdam, 1995).

[2] P. L. Luisi and P. Walde, Giant Vesicles (Perspectives in Supramolecular Chemistry) (Wiley, New York, 2000).

[3] S. R. Keller and R. Skalak, J. Fluid Mech. 120, 27 (1982).

[4] M. Kraus, W. Wintz, U. Seifert, and R. Lipowsky, Phys. Rev. Lett. 77, 3685 (1996).

[5] K. H. de Haas, C. Blom, D. van den Ende, M. H. G. Duits, and J. Mellema, Phys. Rev. E 56, 7132 (1997).
[6] U. Seifert, Eur. Phys. J. B 8, 405 (1999).

[7] T. Biben and C. Misbah, Phys. Rev. E 67, 031908 (2003).

[8] J. Beaucourt, F. Rioual, T. Séon, T. Biben, and C. Misbah, Phys. Rev. E 69, 011906 (2004).

[9] F. Rioual, T. Biben, and C. Misbah, Phys. Rev. E 69, 061914 (2004).

[10] H. Noguchi and G. Gompper, Phys. Rev. Lett. 93, 258102 (2004).

[11] V. Kantsler and V. Steinberg, Phys. Rev. Lett. 95, 258101 (2005). 
[12] V. Kantsler and V. Steinberg, Phys. Rev. Lett. 96, 036001 (2006).

[13] M.-A. Mader, V. Vitkova, M. Abkarian, A. Viallat, and T. Podgorski, Eur. Phys. J. E 19, 389 (2006).

[14] C. Misbah, Phys. Rev. Lett. 96, 028104 (2006).

[15] P. M. Vlahovska and R. S. Gracia, Phys. Rev. E 75, 016313 (2007).

[16] V. V. Lebedev, K. S. Turitsyn, and S. S. Vergeles, Phys. Rev. Lett. 99, 218101 (2007).

[17] G. Danker and C. Misbah, Phys. Rev. Lett. 98, 088104 (2007).

[18] V. Vitkova, M. A. Mader, B. Polack, C. Misbah, and T. Podgorski, Biophys. J. 95, L33 (2008).

[19] J. Deschamps, V. Kantsler, and V. Steinberg, Phys. Rev. Lett. 102, 118105 (2009).

[20] T. Fischer, M. Stohr-Lissen, and H. Schmid-Schonbein, Science 202, 894 (1978).

[21] See EPAPS Document No. E-PLEEE8-80-140911 for movies of the tumbling and vacillating-breathing regimes of a vesicle in a shear flow. For more information on EPAPS, see http:// www.aip.org/pubservs/epaps.html.
[22] H. Noguchi and G. Gompper, Phys. Rev. Lett. 98, 128103 (2007).

[23] G. Danker, T. Biben, T. Podgorski, C. Verdier, and C. Misbah, Phys. Rev. E 76, 041905 (2007).

[24] W. Helfrich, Z. Naturforsch. A 28c, 693 (1973).

[25] Ou-Yang Zhong-can and W. Helfrich, Phys. Rev. A 39, 5280 (1989).

[26] H. Lamb, Hydrodynamics, 6th ed. (Cambridge University Press, Cambridge, England, 1932).

[27] G. Cox, J. Fluid Mech. 37, 601 (1969).

[28] N. A. Frankel and A. Acrivos, J. Fluid Mech. 44, 65 (1970).

[29] D. Barthès-Biesel and J. M. Rallison, J. Fluid Mech. 113, 251 (1981).

[30] S. Kessler, R. Finken, and U. Seifert, J. Fluid Mech. 605, 207 (2008).

[31] G. B. Jeffery, Proc. R. Soc. London, Ser. A 102, 161 (1922).

[32] T. Biben and C. Misbah (unpublished).

[33] M. Abkarian, M. Faivre, and A. Viallat, Phys. Rev. Lett. 98, 188302 (2007) 